Acta Crystallographica Section E

Structure Reports

Online

ISSN 1600-5368

\section{5-Butylamino-2-[2-(dimethylamino)- ethyl]-1H-benz[de]isoquinoline-1,3(2H)- dione}

\section{Li-Juan Xieł}

Institute of Molecular Medicine, Huaqiao University, Quanzhou, Fujian 362021 People's Republic of China.

Correspondence e-mail: xielijuan@hqu.edu.cn

Received 20 April 2010; accepted 19 May 2010

Key indicators: single-crystal X-ray study; $T=293 \mathrm{~K}$; mean $\sigma(\mathrm{C}-\mathrm{C})=0.007 \AA$; $R$ factor $=0.068 ; w R$ factor $=0.175 ;$ data-to-parameter ratio $=15.1$.

The title compound, $\mathrm{C}_{20} \mathrm{H}_{25} \mathrm{~N}_{3} \mathrm{O}_{2}$, is a new amonafide analogue, which exhibits antitumor activity. The asymmetric unit contains two molecules with similar conformations for the substituted aliphatic chains. The two independent molecules form dmers through $\mathrm{N}-\mathrm{H} \cdots \mathrm{N}$ hydrogen bonds. The crystal structure is stabilized via $\pi-\pi$ stacking interactions, the shortest centroid-centroid separation between six-membered rings being 3.673 (2) A.

\section{Related literature}

For general background to amonafide and its antitumour activity, see: Braña et al. (1981, 2001); Braña \& Ramos (2001); Ratain et al. (1991, 1993). For the synthesis of amonafide analogues, see: Xie et al. (2009).

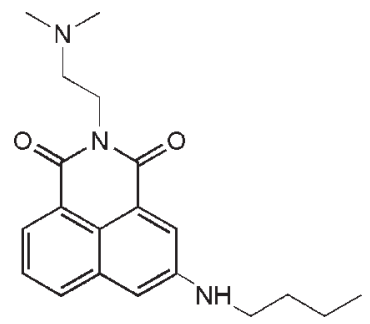

\section{Experimental}

Crystal data

$\mathrm{C}_{20} \mathrm{H}_{25} \mathrm{~N}_{3} \mathrm{O}_{2}$

$M_{r}=339.43$

Triclinic, $P \overline{1}$

$$
\begin{aligned}
& \alpha=72.329(2)^{\circ} \\
& \beta=70.599(2)^{\circ} \\
& \gamma=70.759(2)^{\circ} \\
& V=1816.1(3) \AA^{3} \\
& Z=4
\end{aligned}
$$

Data collection

Bruker SMART APEX CCD areadetector diffractometer

Absorption correction: multi-scan (SADABS; Bruker, 2001)

$T_{\min }=0.739, T_{\max }=1.000$

\section{Refinement}

$R\left[F^{2}>2 \sigma\left(F^{2}\right)\right]=0.068$

$w R\left(F^{2}\right)=0.175$

$S=0.86$

7018 reflections

466 parameters

3 restraints
Mo $K \alpha$ radiation

$\mu=0.08 \mathrm{~mm}^{-1}$

$T=293 \mathrm{~K}$

$0.36 \times 0.33 \times 0.08 \mathrm{~mm}$

10049 measured reflections 7018 independent reflections 3025 reflections with $I>2 \sigma(I)$ $R_{\text {int }}=0.059$

Table 1

Hydrogen-bond geometry $\left(\AA{ }^{\circ}\right)$.

\begin{tabular}{lllll}
\hline$D-\mathrm{H} \cdots A$ & $D-\mathrm{H}$ & $\mathrm{H} \cdots A$ & $D \cdots A$ & $D-\mathrm{H} \cdots A$ \\
\hline $\mathrm{N} 2-\mathrm{H} 2 \cdots \mathrm{N} 6$ & $0.86(2)$ & $2.33(2)$ & $3.176(5)$ & $173(3)$ \\
$\mathrm{N} 5-\mathrm{H} 5 \cdots \mathrm{N} 3$ & $0.85(2)$ & $2.37(2)$ & $3.220(5)$ & $172(4)$ \\
\hline
\end{tabular}

Data collection: SMART (Bruker, 2001); cell refinement: SAINT (Bruker, 2001); data reduction: $S A I N T$; $\operatorname{program}(\mathrm{s})$ used to solve structure: SHELXS97 (Sheldrick, 2008); program(s) used to refine structure: SHELXL97 (Sheldrick, 2008); molecular graphics: SHELXTL (Sheldrick, 2008); software used to prepare material for publication: SHELXL97.

The author is grateful for the support of the Scientific Research Foundation of Huaqiao University (09BS406) and the State Key Laboratory of Oncology in South China.

Supplementary data and figures for this paper are available from the IUCr electronic archives (Reference: BH2282).

\title{
References
}

Braña, M. F., Cacho, M., Gradillas, A., De Pascual-Teresa, B. \& Ramos, A. (2001). Curr. Pharm. Des. 7, 1745-1780.

Braña, M. F. \& Ramos, A. (2001). Curr. Med. Chem. Anti-Cancer Agents, 1, 237-255.

Braña, M. F., Sanz, A. M., Castellano, J. M., Roldan, C. M. \& Roldan, C. (1981). Eur. J. Med. Chem. 16, 207-212.

Bruker (2001). SMART, SAINT and SADABS. Bruker AXS Inc., Madison, Wisconsin, USA.

Ratain, M. J., Mick, R., Berezin, F., Janisch, L., Schilsky, R. L., Vogelzang, N. J. \& Lane, L. B. (1993). Cancer Res. 53, 2304-2308.

Ratain, M. J., Mick, R., Berezin, F., Janisch, L., Schilsky, R. L., Williams, S. F. \& Smiddy, J. (1991). Clin. Pharmacol. Ther. 50, 573-579.

Sheldrick, G. M. (2008). Acta Cryst. A64, 112-122.

Xie, L., Xu, Y., Wang, F., Liu, J., Qian, X. \& Cui, J. (2009). Bioorg. Med. Chem. 17, 804-810. 


\section{supporting information}

Acta Cryst. (2010). E66, o1454 [https://doi.org/10.1107/S1600536810018702]

\section{5-Butylamino-2-[2-(dimethylamino)ethyl]-1 H-benz[de]isoquinoline-1,3(2H)- dione}

\section{Li-Juan Xie}

\section{S1. Comment}

Amonafide (Braña et al., 1981, 2001; Braña \& Ramos, 2001) was the first compound of the naphthalimide family that reached the clinical trial stage and exhibited excellent antitumour activity against advanced breast cancer. However, in the clinical studies, it was found that amonafide was easily metabolized to $N$-acetyl-amonafide by enzyme $N$-acetyltransferase, which caused a high-variable, unpredictable toxicity (Ratain et al., 1991, 1993). In order to reduce the unpredictable toxic effect of the amonafide, we synthesized a series of amonafide analogues (Xie et al., 2009) involved the title compound, which is being reported in this article.

The molecular structure of the title compound is shown in Fig. 1. The asymmetric unit contains two independent molecules, and it is observed that the butyl chains do not present the common all-trans conformation. This uncommon feature could be attributed to formation of intermolecular $\mathrm{N}-\mathrm{H} \cdots \mathrm{N}$ hydrogen bonds in the asymmetric unit [N2 $\cdots \mathrm{N} 6$ and N3 ‥N5], which involve the butyl and the dimethylamino groups, and reduce the intermolecular hindrance. The crystal structure is stabilized via $\pi$ - $\pi$ stacking interactions [centroid-centroid separations: 3.673 (2) and 3.693 (2) $\AA$ ] and intermolecular $\mathrm{N}-\mathrm{H} \cdots \mathrm{N}$ hydrogen bonds, which lead to a supramolecular network of stacked molecules in 1D chains

(Fig. 2). Apart from the functional groups butylamino and $N, N$-dimethylamino-ethylamino, the central 1,8-naphthalimide fused rings system is almost planar.

\section{S2. Experimental}

A mixture of 3-bromide-1,8-naphthalic anhydride $(277 \mathrm{mg}, 1.0 \mathrm{mmol})$ and $N, N$-dimethylethyldiamine $(92 \mathrm{mg}, 1.0 \mathrm{mmol})$ was refluxed in EtOH $(15 \mathrm{ml})$ for $2 \mathrm{~h}$, to give the intermediate 5-bromo-2-[2-(dimethylamino)ethyl]-1H-benz[de]isoquinoline-1,3(2H)-dione. This intermediate (174 mg, $0.5 \mathrm{mmol}), \mathrm{CuI}(9 \mathrm{mg}, 0.05 \mathrm{mmol})$, proline (11 mg, $0.1 \mathrm{mmol})$, $\mathrm{Cs}_{2} \mathrm{CO}_{3}(244 \mathrm{mg}, 0.75 \mathrm{mmol})$ and $n$-butylamine $(0.75 \mathrm{mmol})$ in dry DMSO $(2 \mathrm{ml})$ were mixed and stirred at $383 \mathrm{~K}$ for 9 $\mathrm{h}$ under nitrogen. The crude products were purified by chromatography on silica gel with a mixture of $\mathrm{CH}_{2} \mathrm{Cl}_{2}$ and $\mathrm{MeOH}$ as eluent. Single crystals of the title compound were obtained from a $\mathrm{CH}_{2} \mathrm{Cl}_{2}-\mathrm{MeOH}$ solution.

\section{S3. Refinement}

$\mathrm{C}$-bonded $\mathrm{H}$ atoms were positioned geometrically and refined as riding atoms, with $\mathrm{C}-\mathrm{H}=0.93$ (aromatic), 0.97 (methylene) or $0.96 \AA$ (methyl). Isotropic displacement parameters were computed as $U_{\text {iso }}(\mathrm{H})=1.2 U_{\text {eq }}$ (carrier C) for methylene and aromatic $\mathrm{H}$ atoms, and $U_{\mathrm{iso}}(\mathrm{H})=1.5 U_{\text {eq }}$ (carrier C) for methyl groups. Amine $\mathrm{H}$ atoms $\mathrm{H} 2$ and $\mathrm{H} 5$ were found in a difference map and refined freely. 


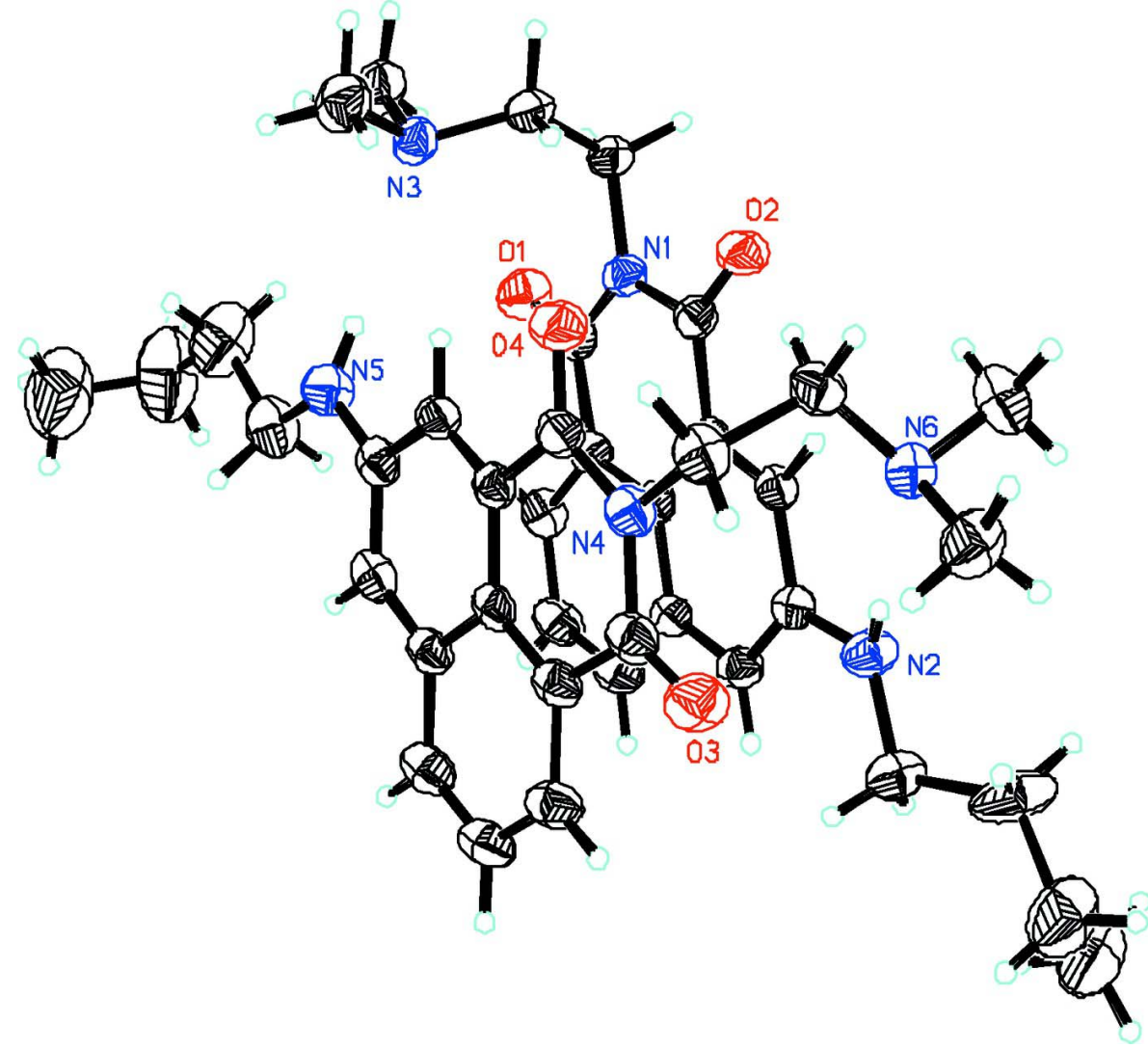

\section{Figure 1}

The structure of the title compound showing $30 \%$ probability displacement ellipsoids and the atom labeling scheme. $\mathrm{H}$ atoms are omitted for clarity.

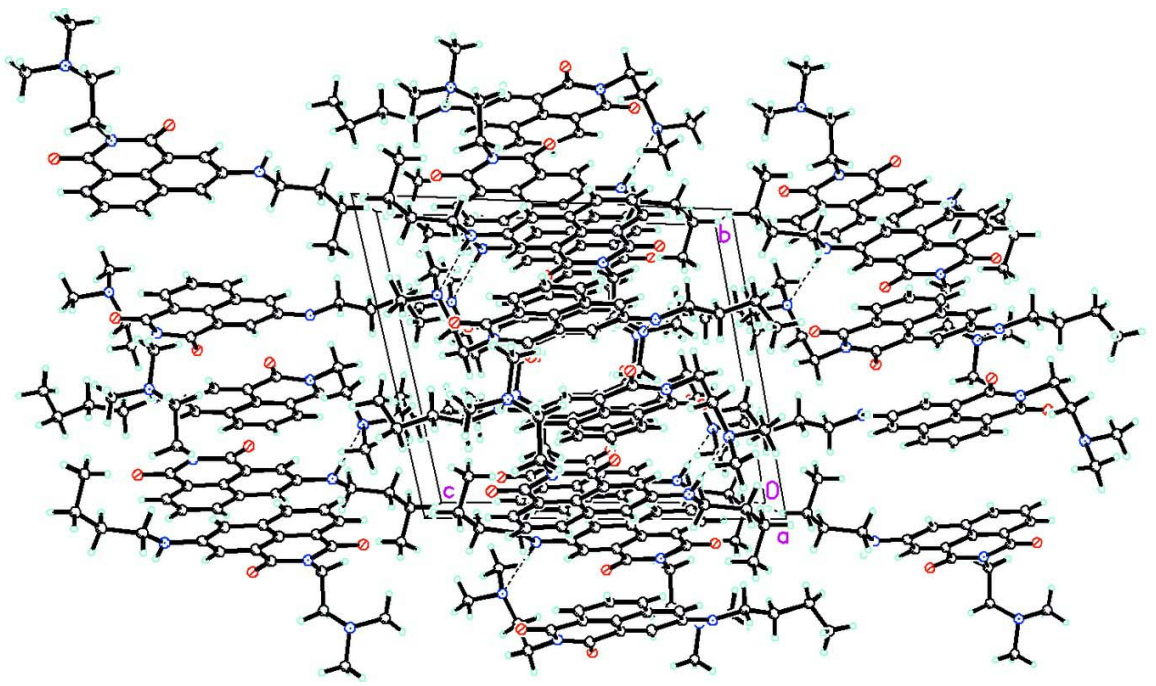

Figure 2

Packing diagram with $\mathrm{H}$ bonds indicated by dashed lines. 
5-Butylamino-2-[2-(dimethylamino)ethyl]-1H-benz[de]isoquinoline- 1,3(2H)-dione

Crystal data

$\mathrm{C}_{20} \mathrm{H}_{25} \mathrm{~N}_{3} \mathrm{O}_{2}$

$M_{r}=339.43$

Triclinic, $P \overline{1}$

Hall symbol: -P 1

$a=11.5978(12) \AA$

$b=12.5362(13) \AA$

$c=14.3721(16) \AA$

$\alpha=72.329(2)^{\circ}$

$\beta=70.599(2)^{\circ}$

$\gamma=70.759(2)^{\circ}$

$V=1816.1(3) \AA^{3}$

Data collection

Bruker SMART APEX CCD area-detector diffractometer

Radiation source: fine-focus sealed tube

Graphite monochromator

$\varphi$ and $\omega$ scans

Absorption correction: multi-scan

(SADABS; Bruker, 2001)

$T_{\min }=0.739, T_{\max }=1.000$

\section{Refinement}

Refinement on $F^{2}$

Least-squares matrix: full

$R\left[F^{2}>2 \sigma\left(F^{2}\right)\right]=0.068$

$w R\left(F^{2}\right)=0.175$

$S=0.86$

7018 reflections

466 parameters

3 restraints

0 constraints

Primary atom site location: structure-invariant direct methods

Secondary atom site location: difference Fourier map
$Z=4$

$F(000)=728$

$D_{\mathrm{x}}=1.241 \mathrm{Mg} \mathrm{m}^{-3}$

Mo $K \alpha$ radiation, $\lambda=0.71073 \AA$

Cell parameters from 1325 reflections

$\theta=5.2-49.0^{\circ}$

$\mu=0.08 \mathrm{~mm}^{-1}$

$T=293 \mathrm{~K}$

Prismatic, yellow

$0.36 \times 0.33 \times 0.08 \mathrm{~mm}$

10049 measured reflections

7018 independent reflections

3025 reflections with $I>2 \sigma(I)$

$R_{\text {int }}=0.059$

$\theta_{\text {max }}=26.0^{\circ}, \theta_{\min }=1.9^{\circ}$

$h=-14 \rightarrow 13$

$k=-15 \rightarrow 11$

$l=-17 \rightarrow 16$

Hydrogen site location: inferred from neighbouring sites

$\mathrm{H}$ atoms treated by a mixture of independent and constrained refinement

$w=1 /\left[\sigma^{2}\left(F_{0}^{2}\right)+(0.0653 P)^{2}\right]$ where $P=\left(F_{\mathrm{o}}^{2}+2 F_{\mathrm{c}}^{2}\right) / 3$

$(\Delta / \sigma)_{\max }<0.001$

$\Delta \rho_{\max }=0.34 \mathrm{e} \AA^{-3}$

$\Delta \rho_{\min }=-0.25$ e $\AA^{-3}$

Extinction correction: SHELXL97 (Sheldrick, 2008), $\mathrm{Fc}^{*}=\mathrm{kFc}\left[1+0.001 \mathrm{xFc}^{2} \lambda^{3} / \sin (2 \theta)\right]^{-1 / 4}$

Extinction coefficient: 0.0040 (10)

Fractional atomic coordinates and isotropic or equivalent isotropic displacement parameters $\left(\AA^{2}\right)$

\begin{tabular}{lllll}
\hline & $x$ & $y$ & $z$ & $U_{\text {iso }} * / U_{\text {eq }}$ \\
\hline O1 & $0.6424(3)$ & $0.8935(2)$ & $0.2061(2)$ & $0.0695(9)$ \\
O2 & $0.4583(2)$ & $0.7887(2)$ & $0.54144(19)$ & $0.0625(8)$ \\
O3 & $0.8585(3)$ & $0.6222(3)$ & $0.8127(2)$ & $0.0754(9)$ \\
O4 & $0.6754(3)$ & $0.5191(3)$ & $0.6307(2)$ & $0.0700(9)$ \\
N1 & $0.5553(3)$ & $0.8357(3)$ & $0.3739(2)$ & $0.0481(8)$ \\
N2 & $0.6845(3)$ & $0.9030(3)$ & $0.7247(3)$ & $0.0592(10)$ \\
N3 & $0.6185(3)$ & $0.6109(3)$ & $0.2983(2)$ & $0.0537(9)$ \\
N4 & $0.7643(3)$ & $0.5748(3)$ & $0.7207(2)$ & $0.0518(8)$ \\
N5 & $0.8927(4)$ & $0.6479(3)$ & $0.2640(3)$ & $0.0665(11)$ \\
N6 & $0.5323(3)$ & $0.7148(3)$ & $0.8571(2)$ & $0.0537(9)$
\end{tabular}




\begin{tabular}{|c|c|c|c|c|}
\hline $\mathrm{C} 1$ & 0.6412 (4) & $0.8876(3)$ & $0.2925(3)$ & $0.0508(10)$ \\
\hline $\mathrm{C} 2$ & $0.5400(4)$ & 0.8308 (3) & $0.4763(3)$ & $0.0480(10)$ \\
\hline $\mathrm{C} 3$ & $0.6272(3)$ & $0.8762(3)$ & $0.4991(3)$ & $0.0394(9)$ \\
\hline $\mathrm{C} 4$ & 0.7179 (3) & 0.9258 (3) & $0.4200(3)$ & $0.0416(9)$ \\
\hline $\mathrm{C} 5$ & 0.7264 (3) & $0.9327(3)$ & 0.3189 (3) & $0.0461(10)$ \\
\hline C6 & $0.8156(4)$ & $0.9811(3)$ & $0.2421(3)$ & $0.0563(11)$ \\
\hline H6 & 0.8212 & 0.9855 & 0.1750 & $0.068^{*}$ \\
\hline $\mathrm{C} 7$ & 0.8974 (4) & $1.0234(4)$ & $0.2660(3)$ & $0.0628(12)$ \\
\hline H7 & 0.9573 & 1.0563 & 0.2144 & $0.075^{*}$ \\
\hline $\mathrm{C} 8$ & $0.8902(3)$ & $1.0169(3)$ & $0.3642(3)$ & $0.0578(11)$ \\
\hline H8 & 0.9458 & 1.0452 & 0.3784 & $0.069^{*}$ \\
\hline C9 & 0.8004 (3) & $0.9683(3)$ & 0.4447 (3) & $0.0453(10)$ \\
\hline $\mathrm{C} 10$ & 0.7902 (3) & $0.9603(3)$ & $0.5474(3)$ & $0.0510(10)$ \\
\hline H10 & 0.8439 & 0.9890 & 0.5635 & $0.061 *$ \\
\hline C11 & $0.7023(3)$ & 0.9107 (3) & 0.6237 (3) & $0.0453(10)$ \\
\hline $\mathrm{C} 12$ & 0.6205 (3) & $0.8696(3)$ & 0.5963 (3) & $0.0455(10)$ \\
\hline H12 & 0.5599 & 0.8368 & 0.6472 & $0.055^{*}$ \\
\hline C13 & 0.7608 (4) & 0.9397 (4) & $0.7643(3)$ & 0.0675 (13) \\
\hline H13A & 0.7753 & 1.0137 & 0.7232 & $0.081 *$ \\
\hline H13B & 0.8422 & 0.8837 & 0.7618 & $0.081 *$ \\
\hline $\mathrm{C} 14$ & $0.6929(5)$ & $0.9512(5)$ & 0.8749 (3) & $0.108(2)$ \\
\hline $\mathrm{H} 14 \mathrm{~A}$ & 0.6063 & 0.9957 & 0.8775 & $0.130^{*}$ \\
\hline H14B & 0.6903 & 0.8744 & 0.9163 & $0.130 *$ \\
\hline $\mathrm{C} 15$ & $0.7481(6)$ & $1.0049(6)$ & $0.9201(4)$ & $0.123(2)$ \\
\hline H15A & 0.8336 & 0.9585 & 0.9202 & $0.148^{*}$ \\
\hline H15B & 0.7003 & 1.0024 & 0.9901 & $0.148^{*}$ \\
\hline $\mathrm{C} 16$ & $0.7529(6)$ & $1.1257(5)$ & 0.8708 (4) & $0.129(2)$ \\
\hline H16A & 0.8098 & 1.1282 & 0.8046 & $0.194^{*}$ \\
\hline H16B & 0.7821 & 1.1557 & 0.9105 & $0.194^{*}$ \\
\hline $\mathrm{H} 16 \mathrm{C}$ & 0.6700 & 1.1718 & 0.8654 & $0.194 *$ \\
\hline $\mathrm{C} 17$ & 0.4680 (4) & 0.7897 (3) & $0.3524(3)$ & $0.0567(11)$ \\
\hline H17A & 0.4649 & 0.8224 & 0.2825 & $0.068^{*}$ \\
\hline H17B & 0.3838 & 0.8145 & 0.3948 & $0.068^{*}$ \\
\hline C18 & $0.5044(4)$ & $0.6589(3)$ & 0.3698 (3) & $0.0572(11)$ \\
\hline H18A & 0.5162 & 0.6266 & 0.4375 & $0.069 *$ \\
\hline H18B & 0.4349 & 0.6345 & 0.3667 & $0.069 *$ \\
\hline C19 & $0.6511(4)$ & $0.4846(4)$ & $0.3333(3)$ & $0.0811(14)$ \\
\hline H19A & 0.5835 & 0.4551 & 0.3354 & $0.122 *$ \\
\hline H19B & 0.6640 & 0.4649 & 0.3997 & $0.122 *$ \\
\hline $\mathrm{H} 19 \mathrm{C}$ & 0.7271 & 0.4513 & 0.2877 & $0.122 *$ \\
\hline $\mathrm{C} 20$ & 0.5971 (4) & $0.6400(4)$ & 0.1975 (3) & $0.0798(14)$ \\
\hline $\mathrm{H} 20 \mathrm{~A}$ & 0.5241 & 0.6167 & 0.2020 & $0.120^{*}$ \\
\hline H20B & 0.6697 & 0.6004 & 0.1532 & $0.120^{*}$ \\
\hline $\mathrm{H} 20 \mathrm{C}$ & 0.5835 & 0.7221 & 0.1714 & $0.120^{*}$ \\
\hline $\mathrm{C} 21$ & 0.8560 (4) & $0.6183(4)$ & $0.7304(3)$ & $0.0565(11)$ \\
\hline $\mathrm{C} 22$ & $0.7540(4)$ & $0.5639(3)$ & $0.6306(3)$ & $0.0529(11)$ \\
\hline $\mathrm{C} 23$ & 0.8405 (3) & $0.6098(3)$ & $0.5360(3)$ & $0.0464(10)$ \\
\hline C24 & $0.9353(3)$ & $0.6535(3)$ & $0.5412(3)$ & $0.0468(10)$ \\
\hline
\end{tabular}




\begin{tabular}{|c|c|c|c|c|}
\hline $\mathrm{C} 25$ & $0.9461(3)$ & $0.6572(3)$ & $0.6348(3)$ & $0.0524(11)$ \\
\hline $\mathrm{C} 26$ & $1.0414(4)$ & 0.6991 (4) & $0.6382(3)$ & $0.0631(12)$ \\
\hline H26 & 1.0481 & 0.7028 & 0.6998 & $0.076^{*}$ \\
\hline $\mathrm{C} 27$ & $1.1271(4)$ & 0.7359 (4) & $0.5481(4)$ & 0.0669 (13) \\
\hline $\mathrm{H} 27$ & 1.1913 & 0.7631 & 0.5505 & $0.080^{*}$ \\
\hline $\mathrm{C} 28$ & $1.1178(4)$ & $0.7325(4)$ & $0.4568(3)$ & $0.0646(12)$ \\
\hline $\mathrm{H} 28$ & 1.1761 & 0.7570 & 0.3981 & $0.078^{*}$ \\
\hline C29 & $1.0213(3)$ & $0.6923(3)$ & $0.4503(3)$ & $0.0508(10)$ \\
\hline C 30 & 1.0069 (4) & 0.6899 (3) & $0.3573(3)$ & 0.0580 \\
\hline $\mathrm{H} 30$ & 1.0636 & 0.7150 & 0.2976 & $0.070^{*}$ \\
\hline $\mathrm{C} 31$ & $0.9108(4)$ & $0.6512(3)$ & $0.3526(3)$ & $0.0520(10)$ \\
\hline C32 & 0.8288 (3) & 0.6093 (3) & $0.4460(3)$ & $0.0513(10)$ \\
\hline H32 & 0.7651 & 0.5807 & 0.4447 & $0.062 *$ \\
\hline $\mathrm{C} 33$ & $0.9693(4)$ & 0.6911 (4) & $0.1663(3)$ & $0.0804(15)$ \\
\hline H33A & 1.0576 & 0.6533 & 0.1636 & $0.097 *$ \\
\hline H33B & 0.9576 & 0.7734 & 0.1576 & $0.097 *$ \\
\hline C34 & $0.9377(6)$ & $0.6714(5)$ & $0.0831(4)$ & $0.119(2)$ \\
\hline $\mathrm{H} 34 \mathrm{~A}$ & 0.8494 & 0.7099 & 0.0861 & $0.142 *$ \\
\hline H34B & 0.9478 & 0.5891 & 0.0931 & $0.142 *$ \\
\hline $\mathrm{C} 35$ & $1.0166(7)$ & $0.7137(6)$ & $-0.0212(4)$ & $0.157(3)$ \\
\hline $\mathrm{H} 35 \mathrm{~A}$ & 0.9636 & 0.7421 & -0.0680 & $0.188^{*}$ \\
\hline H35B & 1.0436 & 0.7785 & -0.0196 & $0.188^{*}$ \\
\hline C36 & $1.1246(7)$ & $0.6296(7)$ & $-0.0586(6)$ & 0.207 (4) \\
\hline $\mathrm{H} 36 \mathrm{~A}$ & 1.1944 & 0.6293 & -0.0367 & $0.310 *$ \\
\hline H36B & 1.1453 & 0.6476 & -0.1311 & $0.310^{*}$ \\
\hline $\mathrm{H} 36 \mathrm{C}$ & 1.1075 & 0.5546 & -0.0334 & $0.310^{*}$ \\
\hline C37 & 0.6757 (4) & $0.5292(3)$ & $0.8135(3)$ & $0.0606(12)$ \\
\hline H37A & 0.7080 & 0.5170 & 0.8711 & $0.073 *$ \\
\hline H37B & 0.6716 & 0.4546 & 0.8096 & $0.073^{*}$ \\
\hline C38 & $0.5425(4)$ & 0.6093 (4) & $0.8305(3)$ & $0.0581(11)$ \\
\hline H38A & 0.5146 & 0.6291 & 0.7694 & $0.070^{*}$ \\
\hline $\mathrm{H} 38 \mathrm{~B}$ & 0.4858 & 0.5677 & 0.8840 & $0.070^{*}$ \\
\hline C39 & $0.4064(4)$ & 0.7929 (4) & $0.8558(3)$ & $0.0811(15)$ \\
\hline H39A & 0.3898 & 0.8027 & 0.7921 & $0.122 *$ \\
\hline H39B & 0.4033 & 0.8667 & 0.8648 & $0.122^{*}$ \\
\hline $\mathrm{H} 39 \mathrm{C}$ & 0.3438 & 0.7604 & 0.9095 & $0.122^{*}$ \\
\hline $\mathrm{C} 40$ & $0.5512(4)$ & $0.6922(4)$ & 0.9577 (3) & $0.0810(15)$ \\
\hline $\mathrm{H} 40 \mathrm{~A}$ & 0.5368 & 0.7644 & 0.9754 & $0.121 *$ \\
\hline $\mathrm{H} 40 \mathrm{~B}$ & 0.6362 & 0.6476 & 0.9576 & $0.121^{*}$ \\
\hline $\mathrm{H} 40 \mathrm{C}$ & 0.4930 & 0.6499 & 1.0062 & $0.121^{*}$ \\
\hline $\mathrm{H} 2$ & $0.649(3)$ & $0.850(3)$ & $0.763(2)$ & $0.065(14)^{*}$ \\
\hline H5 & $0.818(3)$ & $0.645(4)$ & $0.271(3)$ & $0.082(17)^{*}$ \\
\hline
\end{tabular}

Atomic displacement parameters $\left(\AA^{2}\right)$

\begin{tabular}{lllllll}
\hline & $U^{11}$ & $U^{22}$ & $U^{33}$ & $U^{12}$ & $U^{13}$ & $U^{23}$ \\
\hline O1 & $0.087(2)$ & $0.081(2)$ & $0.0454(18)$ & $-0.0301(18)$ & $-0.0225(16)$ & $-0.0058(15)$ \\
O2 & $0.0622(18)$ & $0.076(2)$ & $0.0559(18)$ & $-0.0390(17)$ & $-0.0017(14)$ & $-0.0159(15)$
\end{tabular}




\begin{tabular}{|c|c|c|c|c|c|c|}
\hline $\mathrm{O} 3$ & $0.075(2)$ & $0.097(3)$ & $0.059(2)$ & $-0.0247(19)$ & $-0.0253(17)$ & $-0.0121(17)$ \\
\hline $\mathrm{O} 4$ & $0.0651(19)$ & $0.080(2)$ & $0.073(2)$ & $-0.0406(18)$ & $-0.0019(15)$ & $-0.0221(16)$ \\
\hline N1 & $0.0513(19)$ & $0.042(2)$ & $0.054(2)$ & $-0.0131(16)$ & $-0.0176(16)$ & $-0.0100(16)$ \\
\hline $\mathrm{N} 2$ & $0.070(2)$ & $0.063(3)$ & $0.052(2)$ & $-0.032(2)$ & -0.0168 (19) & $-0.0054(19)$ \\
\hline N3 & $0.058(2)$ & $0.054(2)$ & $0.053(2)$ & $-0.0076(18)$ & $-0.0205(17)$ & $-0.0165(17)$ \\
\hline N4 & $0.049(2)$ & $0.050(2)$ & $0.050(2)$ & $-0.0138(17)$ & $-0.0090(16)$ & $-0.0056(16)$ \\
\hline N5 & $0.068(3)$ & $0.079(3)$ & $0.051(2)$ & $-0.025(2)$ & $-0.010(2)$ & $-0.0106(19)$ \\
\hline N6 & $0.057(2)$ & $0.050(2)$ & $0.048(2)$ & $-0.0141(18)$ & $-0.0105(16)$ & $-0.0056(16)$ \\
\hline $\mathrm{C} 1$ & $0.055(2)$ & $0.042(2)$ & $0.051(3)$ & $-0.009(2)$ & $-0.016(2)$ & $-0.005(2)$ \\
\hline $\mathrm{C} 2$ & $0.048(2)$ & $0.040(2)$ & 0.054 & $-0.012(2)$ & $-0.011(2)$ & $-0.0103(19)$ \\
\hline $\mathrm{C} 3$ & $0.040(2)$ & $0.030(2)$ & $0.045(2)$ & $-0.0054(17)$ & $-0.0114(17)$ & $-0.0080(17)$ \\
\hline $\mathrm{C} 4$ & $0.040(2)$ & $0.031(2)$ & $0.047(2)$ & $-0.0052(17)$ & $-0.0103(18)$ & $-0.0046(17)$ \\
\hline $\mathrm{C} 5$ & $0.047(2)$ & $0.038(2)$ & $0.048(2)$ & $-0.0086(19)$ & $-0.0135(19)$ & $-0.0034(18)$ \\
\hline C6 & $0.057(3)$ & $0.057(3)$ & $0.044(2)$ & $-0.015(2)$ & $-0.009(2)$ & $-0.001(2)$ \\
\hline C7 & $0.051(3)$ & $0.063(3)$ & 0.063 & $-0.025(2)$ & $-0.006(2)$ & $0.004(2)$ \\
\hline C8 & $0.052(3)$ & $0.058(3)$ & $0.061(3)$ & $-0.022(2)$ & $-0.013(2)$ & $-0.003(2)$ \\
\hline C9 & $0.041(2)$ & $0.037(2)$ & $0.052(2)$ & $-0.0097(18)$ & -0.0105 (19) & $-0.0048(18)$ \\
\hline $\mathrm{C} 10$ & $0.051(2)$ & $0.045(3)$ & $0.061(3)$ & $-0.013(2)$ & $-0.020(2)$ & $-0.010(2)$ \\
\hline C11 & $0.048(2)$ & $0.040(2)$ & $0.047(2)$ & $-0.0108(19)$ & $-0.0140(19)$ & $-0.0057(18)$ \\
\hline C12 & $0.043(2)$ & $0.038(2)$ & $0.051(2)$ & $-0.0127(19)$ & $-0.0055(18)$ & $-0.0070(18)$ \\
\hline C13 & $0.080(3)$ & $0.073(3)$ & $0.061(3)$ & $-0.033(3)$ & $-0.026(2)$ & $-0.008(2)$ \\
\hline C14 & $0.153(5)$ & $0.167(6)$ & 0.049 (3) & $-0.115(5)$ & $-0.034(3)$ & $0.005(3)$ \\
\hline C15 & $0.160(6)$ & $0.143(6)$ & $0.069(4)$ & $-0.065(5)$ & $-0.007(4)$ & $-0.024(4)$ \\
\hline C16 & $0.165(6)$ & $0.123(6)$ & $0.108(5)$ & $-0.057(5)$ & $-0.010(4)$ & $-0.044(4)$ \\
\hline C17 & $0.053(2)$ & $0.061(3)$ & $0.065(3)$ & $-0.012(2)$ & $-0.022(2)$ & $-0.021(2)$ \\
\hline $\mathrm{C} 18$ & $0.062(3)$ & $0.060(3)$ & 0.060 & $-0.024(2)$ & $-0.018(2)$ & $-0.016(2)$ \\
\hline C19 & $0.086(3)$ & $0.062(3)$ & 0.097 (4) & $-0.006(3)$ & $-0.037(3)$ & $-0.021(3)$ \\
\hline $\mathrm{C} 20$ & $0.091(3)$ & $0.094(4)$ & $0.065(3)$ & $-0.016(3)$ & $-0.031(3)$ & $-0.029(3)$ \\
\hline $\mathrm{C} 21$ & $0.048(2)$ & $0.051(3)$ & $0.066(3)$ & $-0.006(2)$ & $-0.020(2)$ & $-0.007(2)$ \\
\hline $\mathrm{C} 22$ & $0.045(2)$ & $0.047(3)$ & $0.064(3)$ & $-0.012(2)$ & $-0.008(2)$ & $-0.014(2)$ \\
\hline $\mathrm{C} 23$ & $0.041(2)$ & $0.039(2)$ & $0.054(3)$ & $-0.0058(19)$ & $-0.0110(19)$ & $-0.0098(19)$ \\
\hline $\mathrm{C} 24$ & $0.039(2)$ & $0.033(2)$ & $0.061(3)$ & $0.0009(18)$ & $-0.016(2)$ & $-0.0060(19)$ \\
\hline $\mathrm{C} 25$ & $0.043(2)$ & $0.039(2)$ & $0.070(3)$ & $-0.004(2)$ & $-0.020(2)$ & $-0.005(2)$ \\
\hline $\mathrm{C} 26$ & $0.055(3)$ & $0.057(3)$ & $0.079(3)$ & $-0.011(2)$ & $-0.031(2)$ & $-0.006(2)$ \\
\hline $\mathrm{C} 27$ & $0.048(3)$ & $0.060(3)$ & $0.090(4)$ & $-0.018(2)$ & $-0.022(3)$ & $-0.004(3)$ \\
\hline C28 & $0.048(3)$ & $0.057(3)$ & $0.078(3)$ & $-0.015(2)$ & $-0.015(2)$ & $0.001(2)$ \\
\hline $\mathrm{C} 29$ & $0.040(2)$ & $0.038(2)$ & $0.066(3)$ & $-0.0065(19)$ & $-0.013(2)$ & $-0.004(2)$ \\
\hline $\mathrm{C} 30$ & $0.045(2)$ & $0.049(3)$ & 0.064 & $-0.008(2)$ & $-0.006(2)$ & $-0.003(2)$ \\
\hline C31 & $0.050(2)$ & $0.036(2)$ & $0.060(3)$ & $-0.006(2)$ & $-0.012(2)$ & $-0.004(2)$ \\
\hline C32 & $0.044(2)$ & $0.043(2)$ & $0.067(3)$ & $-0.012(2)$ & $-0.011(2)$ & $-0.014(2)$ \\
\hline C33 & 0.080 & $0.082(4)$ & $0.062(3)$ & $-0.021(3)$ & $-0.005(3)$ & $-0.006(3)$ \\
\hline C34 & $0.163(6)$ & $0.124(5)$ & 0.058 & $-0.052(5)$ & -0.001 (4) & $-0.018(3)$ \\
\hline $\mathrm{C} 35$ & $0.190(7)$ & $0.125(6)$ & $0.086(5)$ & $-0.025(6)$ & $0.024(5)$ & $-0.009(4)$ \\
\hline $\mathrm{C} 36$ & $0.196(9)$ & $0.188(9)$ & $0.203(8)$ & $-0.089(7)$ & $0.074(7)$ & $-0.090(7)$ \\
\hline C37 & $0.063(3)$ & $0.053(3)$ & 0.057 (3) & $-0.019(2)$ & $-0.010(2)$ & $-0.001(2)$ \\
\hline C38 & $0.055(3)$ & $0.063(3)$ & $0.052(3)$ & $-0.025(2)$ & $-0.005(2)$ & $-0.005(2)$ \\
\hline C39 & $0.064(3)$ & $0.074(4)$ & 0.080 & $-0.011(3)$ & $0.000(3)$ & -0.009 (3) \\
\hline $\mathrm{C} 40$ & $0.103(4)$ & $0.092(4)$ & 0.048 & $-0.034(3)$ & $-0.016(3)$ & -0.009 (2) \\
\hline
\end{tabular}


Geometric parameters $\left(\AA,{ }^{\circ}\right)$

\begin{tabular}{|c|c|c|c|}
\hline $\mathrm{O} 1-\mathrm{C} 1$ & $1.216(4)$ & $\mathrm{C} 17-\mathrm{C} 18$ & $1.515(5)$ \\
\hline $\mathrm{O} 2-\mathrm{C} 2$ & $1.222(4)$ & C17-H17A & 0.9700 \\
\hline $\mathrm{O} 3-\mathrm{C} 21$ & $1.209(4)$ & C17-H17B & 0.9700 \\
\hline $\mathrm{O} 4-\mathrm{C} 22$ & $1.218(4)$ & C18-H18A & 0.9700 \\
\hline $\mathrm{N} 1-\mathrm{C} 2$ & $1.406(4)$ & C18-H18B & 0.9700 \\
\hline $\mathrm{N} 1-\mathrm{C} 1$ & $1.406(4)$ & C19-H19A & 0.9600 \\
\hline $\mathrm{N} 1-\mathrm{C} 17$ & $1.469(4)$ & C19-H19B & 0.9600 \\
\hline $\mathrm{N} 2-\mathrm{C} 11$ & $1.375(4)$ & C19-H19C & 0.9600 \\
\hline $\mathrm{N} 2-\mathrm{C} 13$ & $1.442(5)$ & $\mathrm{C} 20-\mathrm{H} 20 \mathrm{~A}$ & 0.9600 \\
\hline $\mathrm{N} 2-\mathrm{H} 2$ & $0.86(2)$ & $\mathrm{C} 20-\mathrm{H} 20 \mathrm{~B}$ & 0.9600 \\
\hline $\mathrm{N} 3-\mathrm{C} 18$ & $1.455(5)$ & $\mathrm{C} 20-\mathrm{H} 20 \mathrm{C}$ & 0.9600 \\
\hline $\mathrm{N} 3-\mathrm{C} 20$ & $1.466(4)$ & $\mathrm{C} 21-\mathrm{C} 25$ & $1.481(5)$ \\
\hline N3-C19 & $1.466(5)$ & $\mathrm{C} 22-\mathrm{C} 23$ & $1.478(5)$ \\
\hline $\mathrm{N} 4-\mathrm{C} 22$ & $1.391(5)$ & $\mathrm{C} 23-\mathrm{C} 32$ & $1.347(5)$ \\
\hline $\mathrm{N} 4-\mathrm{C} 21$ & $1.404(5)$ & $\mathrm{C} 23-\mathrm{C} 24$ & $1.411(5)$ \\
\hline $\mathrm{N} 4-\mathrm{C} 37$ & $1.475(4)$ & $\mathrm{C} 24-\mathrm{C} 25$ & $1.407(5)$ \\
\hline N5-C31 & $1.372(5)$ & $\mathrm{C} 24-\mathrm{C} 29$ & $1.417(5)$ \\
\hline N5-C33 & $1.443(5)$ & $\mathrm{C} 25-\mathrm{C} 26$ & $1.391(5)$ \\
\hline $\mathrm{N} 5-\mathrm{H} 5$ & $0.85(2)$ & $\mathrm{C} 26-\mathrm{C} 27$ & $1.400(5)$ \\
\hline $\mathrm{N} 6-\mathrm{C} 38$ & $1.444(5)$ & $\mathrm{C} 26-\mathrm{H} 26$ & 0.9300 \\
\hline $\mathrm{N} 6-\mathrm{C} 40$ & $1.463(4)$ & $\mathrm{C} 27-\mathrm{C} 28$ & $1.366(5)$ \\
\hline N6-C39 & $1.468(5)$ & $\mathrm{C} 27-\mathrm{H} 27$ & 0.9300 \\
\hline $\mathrm{C} 1-\mathrm{C} 5$ & $1.480(5)$ & $\mathrm{C} 28-\mathrm{C} 29$ & $1.409(5)$ \\
\hline $\mathrm{C} 2-\mathrm{C} 3$ & $1.473(5)$ & $\mathrm{C} 28-\mathrm{H} 28$ & 0.9300 \\
\hline $\mathrm{C} 3-\mathrm{C} 12$ & $1.351(4)$ & $\mathrm{C} 29-\mathrm{C} 30$ & $1.410(5)$ \\
\hline $\mathrm{C} 3-\mathrm{C} 4$ & $1.412(4)$ & $\mathrm{C} 30-\mathrm{C} 31$ & $1.382(5)$ \\
\hline $\mathrm{C} 4-\mathrm{C} 5$ & $1.401(5)$ & $\mathrm{C} 30-\mathrm{H} 30$ & 0.9300 \\
\hline $\mathrm{C} 4-\mathrm{C} 9$ & $1.415(5)$ & $\mathrm{C} 31-\mathrm{C} 32$ & $1.426(5)$ \\
\hline $\mathrm{C} 5-\mathrm{C} 6$ & $1.380(5)$ & $\mathrm{C} 32-\mathrm{H} 32$ & 0.9300 \\
\hline $\mathrm{C} 6-\mathrm{C} 7$ & $1.400(5)$ & $\mathrm{C} 33-\mathrm{C} 34$ & $1.467(6)$ \\
\hline C6-H6 & 0.9300 & $\mathrm{C} 33-\mathrm{H} 33 \mathrm{~A}$ & 0.9700 \\
\hline $\mathrm{C} 7-\mathrm{C} 8$ & $1.365(5)$ & $\mathrm{C} 33-\mathrm{H} 33 \mathrm{~B}$ & 0.9700 \\
\hline $\mathrm{C} 7-\mathrm{H} 7$ & 0.9300 & C34-C35 & $1.514(7)$ \\
\hline $\mathrm{C} 8-\mathrm{C} 9$ & $1.408(5)$ & $\mathrm{C} 34-\mathrm{H} 34 \mathrm{~A}$ & 0.9700 \\
\hline $\mathrm{C} 8-\mathrm{H} 8$ & 0.9300 & $\mathrm{C} 34-\mathrm{H} 34 \mathrm{~B}$ & 0.9700 \\
\hline $\mathrm{C} 9-\mathrm{C} 10$ & $1.416(5)$ & $\mathrm{C} 35-\mathrm{C} 36$ & $1.413(8)$ \\
\hline $\mathrm{C} 10-\mathrm{C} 11$ & $1.375(5)$ & C35-H35A & 0.9700 \\
\hline $\mathrm{C} 10-\mathrm{H} 10$ & 0.9300 & $\mathrm{C} 35-\mathrm{H} 35 \mathrm{~B}$ & 0.9700 \\
\hline $\mathrm{C} 11-\mathrm{C} 12$ & $1.419(5)$ & $\mathrm{C} 36-\mathrm{H} 36 \mathrm{~A}$ & 0.9600 \\
\hline $\mathrm{C} 12-\mathrm{H} 12$ & 0.9300 & $\mathrm{C} 36-\mathrm{H} 36 \mathrm{~B}$ & 0.9600 \\
\hline $\mathrm{C} 13-\mathrm{C} 14$ & $1.550(6)$ & $\mathrm{C} 36-\mathrm{H} 36 \mathrm{C}$ & 0.9600 \\
\hline $\mathrm{C} 13-\mathrm{H} 13 \mathrm{~A}$ & 0.9700 & C37-C38 & $1.527(5)$ \\
\hline C13-H13B & 0.9700 & $\mathrm{C} 37-\mathrm{H} 37 \mathrm{~A}$ & 0.9700 \\
\hline $\mathrm{C} 14-\mathrm{C} 15$ & $1.453(7)$ & C37-H37B & 0.9700 \\
\hline $\mathrm{C} 14-\mathrm{H} 14 \mathrm{~A}$ & 0.9700 & $\mathrm{C} 38-\mathrm{H} 38 \mathrm{~A}$ & 0.9700 \\
\hline C14-H14B & 0.9700 & C $38-H 38 B$ & 0.9700 \\
\hline
\end{tabular}




\begin{tabular}{|c|c|c|c|}
\hline $\mathrm{C} 15-\mathrm{C} 16$ & $1.476(7)$ & C39-H39A & 0.9600 \\
\hline C15-H15A & 0.9700 & C39-H39B & 0.9600 \\
\hline C15-H15B & 0.9700 & $\mathrm{C} 39-\mathrm{H} 39 \mathrm{C}$ & 0.9600 \\
\hline C16-H16A & 0.9600 & $\mathrm{C} 40-\mathrm{H} 40 \mathrm{~A}$ & 0.9600 \\
\hline $\mathrm{C} 16-\mathrm{H} 16 \mathrm{~B}$ & 0.9600 & $\mathrm{C} 40-\mathrm{H} 40 \mathrm{~B}$ & 0.9600 \\
\hline $\mathrm{C} 16-\mathrm{H} 16 \mathrm{C}$ & 0.9600 & $\mathrm{C} 40-\mathrm{H} 40 \mathrm{C}$ & 0.9600 \\
\hline $\mathrm{C} 2-\mathrm{N} 1-\mathrm{C} 1$ & $124.9(3)$ & $\mathrm{N} 3-\mathrm{C} 19-\mathrm{H} 19 \mathrm{C}$ & 109.5 \\
\hline $\mathrm{C} 2-\mathrm{N} 1-\mathrm{C} 17$ & $116.3(3)$ & $\mathrm{H} 19 \mathrm{~A}-\mathrm{C} 19-\mathrm{H} 19 \mathrm{C}$ & 109.5 \\
\hline $\mathrm{C} 1-\mathrm{N} 1-\mathrm{C} 17$ & $118.7(3)$ & $\mathrm{H} 19 \mathrm{~B}-\mathrm{C} 19-\mathrm{H} 19 \mathrm{C}$ & 109.5 \\
\hline $\mathrm{C} 11-\mathrm{N} 2-\mathrm{C} 13$ & $124.1(3)$ & $\mathrm{N} 3-\mathrm{C} 20-\mathrm{H} 20 \mathrm{~A}$ & 109.5 \\
\hline $\mathrm{C} 11-\mathrm{N} 2-\mathrm{H} 2$ & $113(3)$ & N3-C20-H20B & 109.5 \\
\hline $\mathrm{C} 13-\mathrm{N} 2-\mathrm{H} 2$ & $118(3)$ & $\mathrm{H} 20 \mathrm{~A}-\mathrm{C} 20-\mathrm{H} 20 \mathrm{~B}$ & 109.5 \\
\hline $\mathrm{C} 18-\mathrm{N} 3-\mathrm{C} 20$ & $110.6(3)$ & $\mathrm{N} 3-\mathrm{C} 20-\mathrm{H} 20 \mathrm{C}$ & 109.5 \\
\hline $\mathrm{C} 18-\mathrm{N} 3-\mathrm{C} 19$ & $108.1(3)$ & $\mathrm{H} 20 \mathrm{~A}-\mathrm{C} 20-\mathrm{H} 20 \mathrm{C}$ & 109.5 \\
\hline $\mathrm{C} 20-\mathrm{N} 3-\mathrm{C} 19$ & $109.0(3)$ & $\mathrm{H} 20 \mathrm{~B}-\mathrm{C} 20-\mathrm{H} 20 \mathrm{C}$ & 109.5 \\
\hline $\mathrm{C} 22-\mathrm{N} 4-\mathrm{C} 21$ & $125.5(3)$ & $\mathrm{O} 3-\mathrm{C} 21-\mathrm{N} 4$ & $120.5(4)$ \\
\hline $\mathrm{C} 22-\mathrm{N} 4-\mathrm{C} 37$ & $115.9(3)$ & $\mathrm{O} 3-\mathrm{C} 21-\mathrm{C} 25$ & $123.8(4)$ \\
\hline $\mathrm{C} 21-\mathrm{N} 4-\mathrm{C} 37$ & $118.4(3)$ & $\mathrm{N} 4-\mathrm{C} 21-\mathrm{C} 25$ & $115.7(4)$ \\
\hline $\mathrm{C} 31-\mathrm{N} 5-\mathrm{C} 33$ & $122.4(4)$ & $\mathrm{O} 4-\mathrm{C} 22-\mathrm{N} 4$ & $120.6(4)$ \\
\hline $\mathrm{C} 31-\mathrm{N} 5-\mathrm{H} 5$ & $113(3)$ & $\mathrm{O} 4-\mathrm{C} 22-\mathrm{C} 23$ & $122.0(4)$ \\
\hline $\mathrm{C} 33-\mathrm{N} 5-\mathrm{H} 5$ & $120(3)$ & $\mathrm{N} 4-\mathrm{C} 22-\mathrm{C} 23$ & $117.4(4)$ \\
\hline $\mathrm{C} 38-\mathrm{N} 6-\mathrm{C} 40$ & $111.6(3)$ & $\mathrm{C} 32-\mathrm{C} 23-\mathrm{C} 24$ & $120.6(3)$ \\
\hline $\mathrm{C} 38-\mathrm{N} 6-\mathrm{C} 39$ & $109.5(3)$ & $\mathrm{C} 32-\mathrm{C} 23-\mathrm{C} 22$ & $120.1(4)$ \\
\hline $\mathrm{C} 40-\mathrm{N} 6-\mathrm{C} 39$ & $108.7(3)$ & $\mathrm{C} 24-\mathrm{C} 23-\mathrm{C} 22$ & $119.3(4)$ \\
\hline $\mathrm{O} 1-\mathrm{C} 1-\mathrm{N} 1$ & $120.0(4)$ & $\mathrm{C} 25-\mathrm{C} 24-\mathrm{C} 23$ & $121.0(3)$ \\
\hline $\mathrm{O} 1-\mathrm{C} 1-\mathrm{C} 5$ & $123.7(4)$ & $\mathrm{C} 25-\mathrm{C} 24-\mathrm{C} 29$ & $120.2(4)$ \\
\hline $\mathrm{N} 1-\mathrm{C} 1-\mathrm{C} 5$ & $116.3(4)$ & $\mathrm{C} 23-\mathrm{C} 24-\mathrm{C} 29$ & $118.8(4)$ \\
\hline $\mathrm{O} 2-\mathrm{C} 2-\mathrm{N} 1$ & $120.0(4)$ & $\mathrm{C} 26-\mathrm{C} 25-\mathrm{C} 24$ & $120.0(4)$ \\
\hline $\mathrm{O} 2-\mathrm{C} 2-\mathrm{C} 3$ & $123.2(4)$ & $\mathrm{C} 26-\mathrm{C} 25-\mathrm{C} 21$ & $119.2(4)$ \\
\hline $\mathrm{N} 1-\mathrm{C} 2-\mathrm{C} 3$ & $116.9(3)$ & $\mathrm{C} 24-\mathrm{C} 25-\mathrm{C} 21$ & $120.8(4)$ \\
\hline $\mathrm{C} 12-\mathrm{C} 3-\mathrm{C} 4$ & $120.1(3)$ & $\mathrm{C} 25-\mathrm{C} 26-\mathrm{C} 27$ & $119.5(4)$ \\
\hline $\mathrm{C} 12-\mathrm{C} 3-\mathrm{C} 2$ & $119.6(3)$ & $\mathrm{C} 25-\mathrm{C} 26-\mathrm{H} 26$ & 120.3 \\
\hline $\mathrm{C} 4-\mathrm{C} 3-\mathrm{C} 2$ & $120.3(3)$ & $\mathrm{C} 27-\mathrm{C} 26-\mathrm{H} 26$ & 120.3 \\
\hline $\mathrm{C} 5-\mathrm{C} 4-\mathrm{C} 3$ & $120.7(3)$ & $\mathrm{C} 28-\mathrm{C} 27-\mathrm{C} 26$ & $121.1(4)$ \\
\hline $\mathrm{C} 5-\mathrm{C} 4-\mathrm{C} 9$ & $120.5(3)$ & $\mathrm{C} 28-\mathrm{C} 27-\mathrm{H} 27$ & 119.4 \\
\hline $\mathrm{C} 3-\mathrm{C} 4-\mathrm{C} 9$ & $118.7(3)$ & $\mathrm{C} 26-\mathrm{C} 27-\mathrm{H} 27$ & 119.4 \\
\hline $\mathrm{C} 6-\mathrm{C} 5-\mathrm{C} 4$ & $120.3(4)$ & $\mathrm{C} 27-\mathrm{C} 28-\mathrm{C} 29$ & $121.0(4)$ \\
\hline $\mathrm{C} 6-\mathrm{C} 5-\mathrm{C} 1$ & $118.8(4)$ & $\mathrm{C} 27-\mathrm{C} 28-\mathrm{H} 28$ & 119.5 \\
\hline $\mathrm{C} 4-\mathrm{C} 5-\mathrm{C} 1$ & $120.9(3)$ & $\mathrm{C} 29-\mathrm{C} 28-\mathrm{H} 28$ & 119.5 \\
\hline $\mathrm{C} 5-\mathrm{C} 6-\mathrm{C} 7$ & $119.4(4)$ & $\mathrm{C} 28-\mathrm{C} 29-\mathrm{C} 30$ & $122.7(4)$ \\
\hline $\mathrm{C} 5-\mathrm{C} 6-\mathrm{H} 6$ & 120.3 & $\mathrm{C} 28-\mathrm{C} 29-\mathrm{C} 24$ & $118.2(4)$ \\
\hline $\mathrm{C} 7-\mathrm{C} 6-\mathrm{H} 6$ & 120.3 & $\mathrm{C} 30-\mathrm{C} 29-\mathrm{C} 24$ & $119.1(4)$ \\
\hline $\mathrm{C} 8-\mathrm{C} 7-\mathrm{C} 6$ & $120.7(4)$ & $\mathrm{C} 31-\mathrm{C} 30-\mathrm{C} 29$ & $121.7(4)$ \\
\hline $\mathrm{C} 8-\mathrm{C} 7-\mathrm{H} 7$ & 119.6 & $\mathrm{C} 31-\mathrm{C} 30-\mathrm{H} 30$ & 119.1 \\
\hline $\mathrm{C} 6-\mathrm{C} 7-\mathrm{H} 7$ & 119.6 & $\mathrm{C} 29-\mathrm{C} 30-\mathrm{H} 30$ & 119.1 \\
\hline $\mathrm{C} 7-\mathrm{C} 8-\mathrm{C} 9$ & $121.5(4)$ & $\mathrm{N} 5-\mathrm{C} 31-\mathrm{C} 30$ & $123.8(4)$ \\
\hline $\mathrm{C} 7-\mathrm{C} 8-\mathrm{H} 8$ & 119.2 & $\mathrm{~N} 5-\mathrm{C} 31-\mathrm{C} 32$ & $118.8(4)$ \\
\hline
\end{tabular}




\begin{tabular}{|c|c|c|c|}
\hline $\mathrm{C} 9-\mathrm{C} 8-\mathrm{H} 8$ & 119.2 & $\mathrm{C} 30-\mathrm{C} 31-\mathrm{C} 32$ & $117.4(4)$ \\
\hline $\mathrm{C} 8-\mathrm{C} 9-\mathrm{C} 4$ & $117.5(4)$ & $\mathrm{C} 23-\mathrm{C} 32-\mathrm{C} 31$ & $122.3(4)$ \\
\hline $\mathrm{C} 8-\mathrm{C} 9-\mathrm{C} 10$ & $123.0(4)$ & $\mathrm{C} 23-\mathrm{C} 32-\mathrm{H} 32$ & 118.8 \\
\hline $\mathrm{C} 4-\mathrm{C} 9-\mathrm{C} 10$ & $119.6(3)$ & $\mathrm{C} 31-\mathrm{C} 32-\mathrm{H} 32$ & 118.8 \\
\hline $\mathrm{C} 11-\mathrm{C} 10-\mathrm{C} 9$ & $121.1(4)$ & $\mathrm{N} 5-\mathrm{C} 33-\mathrm{C} 34$ & $112.5(4)$ \\
\hline $\mathrm{C} 11-\mathrm{C} 10-\mathrm{H} 10$ & 119.5 & $\mathrm{~N} 5-\mathrm{C} 33-\mathrm{H} 33 \mathrm{~A}$ & 109.1 \\
\hline $\mathrm{C} 9-\mathrm{C} 10-\mathrm{H} 10$ & 119.5 & $\mathrm{C} 34-\mathrm{C} 33-\mathrm{H} 33 \mathrm{~A}$ & 109.1 \\
\hline $\mathrm{C} 10-\mathrm{C} 11-\mathrm{N} 2$ & $124.3(4)$ & $\mathrm{N} 5-\mathrm{C} 33-\mathrm{H} 33 \mathrm{~B}$ & 109.1 \\
\hline $\mathrm{C} 10-\mathrm{C} 11-\mathrm{C} 12$ & $118.0(4)$ & $\mathrm{C} 34-\mathrm{C} 33-\mathrm{H} 33 \mathrm{~B}$ & 109.1 \\
\hline $\mathrm{N} 2-\mathrm{C} 11-\mathrm{C} 12$ & $117.7(3)$ & $\mathrm{H} 33 \mathrm{~A}-\mathrm{C} 33-\mathrm{H} 33 \mathrm{~B}$ & 107.8 \\
\hline $\mathrm{C} 3-\mathrm{C} 12-\mathrm{C} 11$ & $122.6(3)$ & $\mathrm{C} 33-\mathrm{C} 34-\mathrm{C} 35$ & $114.7(5)$ \\
\hline $\mathrm{C} 3-\mathrm{C} 12-\mathrm{H} 12$ & 118.7 & $\mathrm{C} 33-\mathrm{C} 34-\mathrm{H} 34 \mathrm{~A}$ & 108.6 \\
\hline $\mathrm{C} 11-\mathrm{C} 12-\mathrm{H} 12$ & 118.7 & $\mathrm{C} 35-\mathrm{C} 34-\mathrm{H} 34 \mathrm{~A}$ & 108.6 \\
\hline $\mathrm{N} 2-\mathrm{C} 13-\mathrm{C} 14$ & $109.9(3)$ & $\mathrm{C} 33-\mathrm{C} 34-\mathrm{H} 34 \mathrm{~B}$ & 108.6 \\
\hline $\mathrm{N} 2-\mathrm{C} 13-\mathrm{H} 13 \mathrm{~A}$ & 109.7 & C $35-\mathrm{C} 34-\mathrm{H} 34 \mathrm{~B}$ & 108.6 \\
\hline $\mathrm{C} 14-\mathrm{C} 13-\mathrm{H} 13 \mathrm{~A}$ & 109.7 & $\mathrm{H} 34 \mathrm{~A}-\mathrm{C} 34-\mathrm{H} 34 \mathrm{~B}$ & 107.6 \\
\hline $\mathrm{N} 2-\mathrm{C} 13-\mathrm{H} 13 \mathrm{~B}$ & 109.7 & $\mathrm{C} 36-\mathrm{C} 35-\mathrm{C} 34$ & $114.9(6)$ \\
\hline $\mathrm{C} 14-\mathrm{C} 13-\mathrm{H} 13 \mathrm{~B}$ & 109.7 & $\mathrm{C} 36-\mathrm{C} 35-\mathrm{H} 35 \mathrm{~A}$ & 108.5 \\
\hline $\mathrm{H} 13 \mathrm{~A}-\mathrm{C} 13-\mathrm{H} 13 \mathrm{~B}$ & 108.2 & $\mathrm{C} 34-\mathrm{C} 35-\mathrm{H} 35 \mathrm{~A}$ & 108.5 \\
\hline $\mathrm{C} 15-\mathrm{C} 14-\mathrm{C} 13$ & $116.4(4)$ & $\mathrm{C} 36-\mathrm{C} 35-\mathrm{H} 35 \mathrm{~B}$ & 108.5 \\
\hline $\mathrm{C} 15-\mathrm{C} 14-\mathrm{H} 14 \mathrm{~A}$ & 108.2 & $\mathrm{C} 34-\mathrm{C} 35-\mathrm{H} 35 \mathrm{~B}$ & 108.5 \\
\hline $\mathrm{C} 13-\mathrm{C} 14-\mathrm{H} 14 \mathrm{~A}$ & 108.2 & $\mathrm{H} 35 \mathrm{~A}-\mathrm{C} 35-\mathrm{H} 35 \mathrm{~B}$ & 107.5 \\
\hline $\mathrm{C} 15-\mathrm{C} 14-\mathrm{H} 14 \mathrm{~B}$ & 108.2 & $\mathrm{C} 35-\mathrm{C} 36-\mathrm{H} 36 \mathrm{~A}$ & 109.5 \\
\hline $\mathrm{C} 13-\mathrm{C} 14-\mathrm{H} 14 \mathrm{~B}$ & 108.2 & $\mathrm{C} 35-\mathrm{C} 36-\mathrm{H} 36 \mathrm{~B}$ & 109.5 \\
\hline $\mathrm{H} 14 \mathrm{~A}-\mathrm{C} 14-\mathrm{H} 14 \mathrm{~B}$ & 107.3 & $\mathrm{H} 36 \mathrm{~A}-\mathrm{C} 36-\mathrm{H} 36 \mathrm{~B}$ & 109.5 \\
\hline $\mathrm{C} 14-\mathrm{C} 15-\mathrm{C} 16$ & $116.1(5)$ & $\mathrm{C} 35-\mathrm{C} 36-\mathrm{H} 36 \mathrm{C}$ & 109.5 \\
\hline $\mathrm{C} 14-\mathrm{C} 15-\mathrm{H} 15 \mathrm{~A}$ & 108.3 & $\mathrm{H} 36 \mathrm{~A}-\mathrm{C} 36-\mathrm{H} 36 \mathrm{C}$ & 109.5 \\
\hline $\mathrm{C} 16-\mathrm{C} 15-\mathrm{H} 15 \mathrm{~A}$ & 108.3 & $\mathrm{H} 36 \mathrm{~B}-\mathrm{C} 36-\mathrm{H} 36 \mathrm{C}$ & 109.5 \\
\hline $\mathrm{C} 14-\mathrm{C} 15-\mathrm{H} 15 \mathrm{~B}$ & 108.3 & $\mathrm{~N} 4-\mathrm{C} 37-\mathrm{C} 38$ & $113.2(3)$ \\
\hline $\mathrm{C} 16-\mathrm{C} 15-\mathrm{H} 15 \mathrm{~B}$ & 108.3 & $\mathrm{~N} 4-\mathrm{C} 37-\mathrm{H} 37 \mathrm{~A}$ & 108.9 \\
\hline $\mathrm{H} 15 \mathrm{~A}-\mathrm{C} 15-\mathrm{H} 15 \mathrm{~B}$ & 107.4 & C38-C37-H37A & 108.9 \\
\hline $\mathrm{C} 15-\mathrm{C} 16-\mathrm{H} 16 \mathrm{~A}$ & 109.5 & N4-C37-H37B & 108.9 \\
\hline $\mathrm{C} 15-\mathrm{C} 16-\mathrm{H} 16 \mathrm{~B}$ & 109.5 & $\mathrm{C} 38-\mathrm{C} 37-\mathrm{H} 37 \mathrm{~B}$ & 108.9 \\
\hline $\mathrm{H} 16 \mathrm{~A}-\mathrm{C} 16-\mathrm{H} 16 \mathrm{~B}$ & 109.5 & $\mathrm{H} 37 \mathrm{~A}-\mathrm{C} 37-\mathrm{H} 37 \mathrm{~B}$ & 107.8 \\
\hline $\mathrm{C} 15-\mathrm{C} 16-\mathrm{H} 16 \mathrm{C}$ & 109.5 & $\mathrm{~N} 6-\mathrm{C} 38-\mathrm{C} 37$ & $114.1(3)$ \\
\hline $\mathrm{H} 16 \mathrm{~A}-\mathrm{C} 16-\mathrm{H} 16 \mathrm{C}$ & 109.5 & $\mathrm{~N} 6-\mathrm{C} 38-\mathrm{H} 38 \mathrm{~A}$ & 108.7 \\
\hline $\mathrm{H} 16 \mathrm{~B}-\mathrm{C} 16-\mathrm{H} 16 \mathrm{C}$ & 109.5 & C37-C $38-\mathrm{H} 38 \mathrm{~A}$ & 108.7 \\
\hline $\mathrm{N} 1-\mathrm{C} 17-\mathrm{C} 18$ & $113.6(3)$ & N6-C38-H38B & 108.7 \\
\hline $\mathrm{N} 1-\mathrm{C} 17-\mathrm{H} 17 \mathrm{~A}$ & 108.9 & $\mathrm{C} 37-\mathrm{C} 38-\mathrm{H} 38 \mathrm{~B}$ & 108.7 \\
\hline $\mathrm{C} 18-\mathrm{C} 17-\mathrm{H} 17 \mathrm{~A}$ & 108.9 & $\mathrm{H} 38 \mathrm{~A}-\mathrm{C} 38-\mathrm{H} 38 \mathrm{~B}$ & 107.6 \\
\hline $\mathrm{N} 1-\mathrm{C} 17-\mathrm{H} 17 \mathrm{~B}$ & 108.9 & $\mathrm{~N} 6-\mathrm{C} 39-\mathrm{H} 39 \mathrm{~A}$ & 109.5 \\
\hline $\mathrm{C} 18-\mathrm{C} 17-\mathrm{H} 17 \mathrm{~B}$ & 108.9 & $\mathrm{~N} 6-\mathrm{C} 39-\mathrm{H} 39 \mathrm{~B}$ & 109.5 \\
\hline $\mathrm{H} 17 \mathrm{~A}-\mathrm{C} 17-\mathrm{H} 17 \mathrm{~B}$ & 107.7 & $\mathrm{H} 39 \mathrm{~A}-\mathrm{C} 39-\mathrm{H} 39 \mathrm{~B}$ & 109.5 \\
\hline $\mathrm{N} 3-\mathrm{C} 18-\mathrm{C} 17$ & $114.7(3)$ & $\mathrm{N} 6-\mathrm{C} 39-\mathrm{H} 39 \mathrm{C}$ & 109.5 \\
\hline N3-C18-H18A & 108.6 & $\mathrm{H} 39 \mathrm{~A}-\mathrm{C} 39-\mathrm{H} 39 \mathrm{C}$ & 109.5 \\
\hline $\mathrm{C} 17-\mathrm{C} 18-\mathrm{H} 18 \mathrm{~A}$ & 108.6 & $\mathrm{H} 39 \mathrm{~B}-\mathrm{C} 39-\mathrm{H} 39 \mathrm{C}$ & 109.5 \\
\hline N3- $18-\mathrm{H} 18 \mathrm{~B}$ & 108.6 & $\mathrm{~N} 6-\mathrm{C} 40-\mathrm{H} 40 \mathrm{~A}$ & 109.5 \\
\hline $\mathrm{C} 17-\mathrm{C} 18-\mathrm{H} 18 \mathrm{~B}$ & 108.6 & $\mathrm{~N} 6-\mathrm{C} 40-\mathrm{H} 40 \mathrm{~B}$ & 109.5 \\
\hline
\end{tabular}


H18A-C18-H18B

N3-C19-H19A

N3-C19-H19B

H19A-C19-H19B

$\mathrm{C} 2-\mathrm{N} 1-\mathrm{C} 1-\mathrm{O} 1$

$\mathrm{C} 17-\mathrm{N} 1-\mathrm{C} 1-\mathrm{O} 1$

$\mathrm{C} 2-\mathrm{N} 1-\mathrm{C} 1-\mathrm{C} 5$

$\mathrm{C} 17-\mathrm{N} 1-\mathrm{C} 1-\mathrm{C} 5$

$\mathrm{C} 1-\mathrm{N} 1-\mathrm{C} 2-\mathrm{O} 2$

$\mathrm{C} 17-\mathrm{N} 1-\mathrm{C} 2-\mathrm{O} 2$

$\mathrm{C} 1-\mathrm{N} 1-\mathrm{C} 2-\mathrm{C} 3$

$\mathrm{C} 17-\mathrm{N} 1-\mathrm{C} 2-\mathrm{C} 3$

$\mathrm{O} 2-\mathrm{C} 2-\mathrm{C} 3-\mathrm{C} 12$

$\mathrm{N} 1-\mathrm{C} 2-\mathrm{C} 3-\mathrm{C} 12$

$\mathrm{O} 2-\mathrm{C} 2-\mathrm{C} 3-\mathrm{C} 4$

$\mathrm{N} 1-\mathrm{C} 2-\mathrm{C} 3-\mathrm{C} 4$

$\mathrm{C} 12-\mathrm{C} 3-\mathrm{C} 4-\mathrm{C} 5$

$\mathrm{C} 2-\mathrm{C} 3-\mathrm{C} 4-\mathrm{C} 5$

$\mathrm{C} 12-\mathrm{C} 3-\mathrm{C} 4-\mathrm{C} 9$

$\mathrm{C} 2-\mathrm{C} 3-\mathrm{C} 4-\mathrm{C} 9$

$\mathrm{C} 3-\mathrm{C} 4-\mathrm{C} 5-\mathrm{C} 6$

$\mathrm{C} 9-\mathrm{C} 4-\mathrm{C} 5-\mathrm{C} 6$

$\mathrm{C} 3-\mathrm{C} 4-\mathrm{C} 5-\mathrm{C} 1$

$\mathrm{C} 9-\mathrm{C} 4-\mathrm{C} 5-\mathrm{C} 1$

$\mathrm{O} 1-\mathrm{C} 1-\mathrm{C} 5-\mathrm{C} 6$

$\mathrm{N} 1-\mathrm{C} 1-\mathrm{C} 5-\mathrm{C} 6$

$\mathrm{O} 1-\mathrm{C} 1-\mathrm{C} 5-\mathrm{C} 4$

$\mathrm{N} 1-\mathrm{C} 1-\mathrm{C} 5-\mathrm{C} 4$

$\mathrm{C} 4-\mathrm{C} 5-\mathrm{C} 6-\mathrm{C} 7$

$\mathrm{C} 1-\mathrm{C} 5-\mathrm{C} 6-\mathrm{C} 7$

$\mathrm{C} 5-\mathrm{C} 6-\mathrm{C} 7-\mathrm{C} 8$

$\mathrm{C} 6-\mathrm{C} 7-\mathrm{C} 8-\mathrm{C} 9$

$\mathrm{C} 7-\mathrm{C} 8-\mathrm{C} 9-\mathrm{C} 4$

$\mathrm{C} 7-\mathrm{C} 8-\mathrm{C} 9-\mathrm{C} 10$

$\mathrm{C} 5-\mathrm{C} 4-\mathrm{C} 9-\mathrm{C} 8$

$\mathrm{C} 3-\mathrm{C} 4-\mathrm{C} 9-\mathrm{C} 8$

$\mathrm{C} 5-\mathrm{C} 4-\mathrm{C} 9-\mathrm{C} 10$

$\mathrm{C} 3-\mathrm{C} 4-\mathrm{C} 9-\mathrm{C} 10$

$\mathrm{C} 8-\mathrm{C} 9-\mathrm{C} 10-\mathrm{C} 11$

$\mathrm{C} 4-\mathrm{C} 9-\mathrm{C} 10-\mathrm{C} 11$

$\mathrm{C} 9-\mathrm{C} 10-\mathrm{C} 11-\mathrm{N} 2$

$\mathrm{C} 9-\mathrm{C} 10-\mathrm{C} 11-\mathrm{C} 12$

C13-N2-C11- 10

$\mathrm{C} 13-\mathrm{N} 2-\mathrm{C} 11-\mathrm{C} 12$

$\mathrm{C} 4-\mathrm{C} 3-\mathrm{C} 12-\mathrm{C} 11$

$\mathrm{C} 2-\mathrm{C} 3-\mathrm{C} 12-\mathrm{C} 11$

$\mathrm{C} 10-\mathrm{C} 11-\mathrm{C} 12-\mathrm{C} 3$
107.6

109.5

109.5

109.5

$-175.7(3)$

$-0.1(5)$

$4.2(5)$

$179.8(3)$

$176.4(3)$

$0.8(5)$

$-4.5(5)$

$179.8(3)$

$1.6(5)$

$-177.4(3)$

$-178.8(3)$

$2.2(5)$

$179.6(3)$

$0.0(5)$

$-0.2(5)$

$-179.8(3)$

$-179.8(3)$

$0.0(5)$

$-0.3(5)$

179.5 (3)

$-2.2(6)$

$177.9(3)$

$178.3(4)$

$-1.6(5)$

$-0.1(6)$

$-179.6(3)$

$0.3(6)$

-0.4 (6)

$0.2(6)$

$-179.9(4)$

-0.1 (5)

$179.8(3)$

$-179.9(3)$

$-0.1(5)$

$-179.1(4)$

$0.7(5)$

$-178.0(3)$

$-1.1(5)$

$-3.9(6)$

$179.2(4)$

$-0.2(5)$

$179.4(3)$

$0.8(5)$
$\mathrm{H} 40 \mathrm{~A}-\mathrm{C} 40-\mathrm{H} 40 \mathrm{~B}$

$\mathrm{N} 6-\mathrm{C} 40-\mathrm{H} 40 \mathrm{C}$

$\mathrm{H} 40 \mathrm{~A}-\mathrm{C} 40-\mathrm{H} 40 \mathrm{C}$

$\mathrm{H} 40 \mathrm{~B}-\mathrm{C} 40-\mathrm{H} 40 \mathrm{C}$

$\mathrm{C} 22-\mathrm{N} 4-\mathrm{C} 21-\mathrm{O} 3$

$\mathrm{C} 37-\mathrm{N} 4-\mathrm{C} 21-\mathrm{O} 3$

$\mathrm{C} 22-\mathrm{N} 4-\mathrm{C} 21-\mathrm{C} 25$

$\mathrm{C} 37-\mathrm{N} 4-\mathrm{C} 21-\mathrm{C} 25$

$\mathrm{C} 21-\mathrm{N} 4-\mathrm{C} 22-\mathrm{O} 4$

$\mathrm{C} 37-\mathrm{N} 4-\mathrm{C} 22-\mathrm{O} 4$

$\mathrm{C} 21-\mathrm{N} 4-\mathrm{C} 22-\mathrm{C} 23$

$\mathrm{C} 37-\mathrm{N} 4-\mathrm{C} 22-\mathrm{C} 23$

$\mathrm{O} 4-\mathrm{C} 22-\mathrm{C} 23-\mathrm{C} 32$

$\mathrm{N} 4-\mathrm{C} 22-\mathrm{C} 23-\mathrm{C} 32$

$\mathrm{O} 4-\mathrm{C} 22-\mathrm{C} 23-\mathrm{C} 24$

$\mathrm{N} 4-\mathrm{C} 22-\mathrm{C} 23-\mathrm{C} 24$

$\mathrm{C} 32-\mathrm{C} 23-\mathrm{C} 24-\mathrm{C} 25$

$\mathrm{C} 22-\mathrm{C} 23-\mathrm{C} 24-\mathrm{C} 25$

$\mathrm{C} 32-\mathrm{C} 23-\mathrm{C} 24-\mathrm{C} 29$

$\mathrm{C} 22-\mathrm{C} 23-\mathrm{C} 24-\mathrm{C} 29$

$\mathrm{C} 23-\mathrm{C} 24-\mathrm{C} 25-\mathrm{C} 26$

$\mathrm{C} 29-\mathrm{C} 24-\mathrm{C} 25-\mathrm{C} 26$

$\mathrm{C} 23-\mathrm{C} 24-\mathrm{C} 25-\mathrm{C} 21$

$\mathrm{C} 29-\mathrm{C} 24-\mathrm{C} 25-\mathrm{C} 21$

$\mathrm{O} 3-\mathrm{C} 21-\mathrm{C} 25-\mathrm{C} 26$

$\mathrm{N} 4-\mathrm{C} 21-\mathrm{C} 25-\mathrm{C} 26$

$\mathrm{O} 3-\mathrm{C} 21-\mathrm{C} 25-\mathrm{C} 24$

N4-C21-C25-C24

$\mathrm{C} 24-\mathrm{C} 25-\mathrm{C} 26-\mathrm{C} 27$

$\mathrm{C} 21-\mathrm{C} 25-\mathrm{C} 26-\mathrm{C} 27$

$\mathrm{C} 25-\mathrm{C} 26-\mathrm{C} 27-\mathrm{C} 28$

$\mathrm{C} 26-\mathrm{C} 27-\mathrm{C} 28-\mathrm{C} 29$

$\mathrm{C} 27-\mathrm{C} 28-\mathrm{C} 29-\mathrm{C} 30$

$\mathrm{C} 27-\mathrm{C} 28-\mathrm{C} 29-\mathrm{C} 24$

$\mathrm{C} 25-\mathrm{C} 24-\mathrm{C} 29-\mathrm{C} 28$

$\mathrm{C} 23-\mathrm{C} 24-\mathrm{C} 29-\mathrm{C} 28$

$\mathrm{C} 25-\mathrm{C} 24-\mathrm{C} 29-\mathrm{C} 30$

$\mathrm{C} 23-\mathrm{C} 24-\mathrm{C} 29-\mathrm{C} 30$

$\mathrm{C} 28-\mathrm{C} 29-\mathrm{C} 30-\mathrm{C} 31$

$\mathrm{C} 24-\mathrm{C} 29-\mathrm{C} 30-\mathrm{C} 31$

$\mathrm{C} 33-\mathrm{N} 5-\mathrm{C} 31-\mathrm{C} 30$

$\mathrm{C} 33-\mathrm{N} 5-\mathrm{C} 31-\mathrm{C} 32$

$\mathrm{C} 29-\mathrm{C} 30-\mathrm{C} 31-\mathrm{N} 5$

$\mathrm{C} 29-\mathrm{C} 30-\mathrm{C} 31-\mathrm{C} 32$

$\mathrm{C} 24-\mathrm{C} 23-\mathrm{C} 32-\mathrm{C} 31$

$\mathrm{C} 22-\mathrm{C} 23-\mathrm{C} 32-\mathrm{C} 31$

$\mathrm{N} 5-\mathrm{C} 31-\mathrm{C} 32-\mathrm{C} 23$
109.5

109.5

109.5

109.5

$-178.5(4)$

$-2.9(6)$

$1.3(5)$

$177.0(3)$

175.8 (4)

$0.1(5)$

$-4.9(5)$

179.4 (3)

$4.1(6)$

$-175.2(3)$

$-175.7(4)$

$5.0(5)$

$178.3(3)$

$-1.9(5)$

$-2.7(5)$

$177.1(3)$

$179.1(3)$

$0.1(5)$

$-1.8(5)$

$179.2(3)$

$1.1(6)$

-178.7 (3)

-178.0 (4)

$2.1(5)$

$-1.0(6)$

179.9 (4)

$0.8(6)$

0.3 (6)

$178.5(4)$

-1.1 (6)

0.9 (5)

-178.1 (3)

$-178.8(3)$

$2.2(5)$

-179.4 (4)

$0.3(6)$

$-3.5(6)$

178.4 (4)

179.6 (4)

$-2.3(6)$

0.6 (6)

$-179.2(3)$

$-179.9(4)$ 


$\begin{array}{llll}\mathrm{N} 2-\mathrm{C} 11-\mathrm{C} 12-\mathrm{C} 3 & 178.0(3) & \mathrm{C} 30-\mathrm{C} 31-\mathrm{C} 32-\mathrm{C} 23 & 1.9(6) \\ \mathrm{C} 11-\mathrm{N} 2-\mathrm{C} 13-\mathrm{C} 14 & 164.1(4) & \mathrm{C} 31-\mathrm{N} 5-\mathrm{C} 33-\mathrm{C} 34 & 176.3(4) \\ \mathrm{N} 2-\mathrm{C} 13-\mathrm{C} 14-\mathrm{C} 15 & -170.6(5) & \mathrm{N} 5-\mathrm{C} 33-\mathrm{C} 34-\mathrm{C} 35 & -179.2(5) \\ \mathrm{C} 13-\mathrm{C} 14-\mathrm{C} 15-\mathrm{C} 16 & 61.5(8) & \mathrm{C} 33-\mathrm{C} 34-\mathrm{C} 35-\mathrm{C} 36 & 93.2(8) \\ \mathrm{C} 2-\mathrm{N} 1-\mathrm{C} 17-\mathrm{C} 18 & -79.6(4) & \mathrm{C} 22-\mathrm{N} 4-\mathrm{C} 37-\mathrm{C} 38 & -77.6(4) \\ \mathrm{C} 1-\mathrm{N} 1-\mathrm{C} 17-\mathrm{C} 18 & 104.4(4) & \mathrm{C} 21-\mathrm{N} 4-\mathrm{C} 37-\mathrm{C} 38 & 106.3(4) \\ \mathrm{C} 20-\mathrm{N} 3-\mathrm{C} 18-\mathrm{C} 17 & -68.8(4) & \mathrm{C} 40-\mathrm{N} 6-\mathrm{C} 38-\mathrm{C} 37 & -68.5(4) \\ \mathrm{C} 19-\mathrm{N} 3-\mathrm{C} 18-\mathrm{C} 17 & 172.0(3) & \mathrm{C} 39-\mathrm{N} 6-\mathrm{C} 38-\mathrm{C} 37 & 171.0(3) \\ \mathrm{N} 1-\mathrm{C} 17-\mathrm{C} 18-\mathrm{N} 3 & -69.1(4) & \mathrm{N} 4-\mathrm{C} 37-\mathrm{C} 38-\mathrm{N} 6 & -70.1(4)\end{array}$

Hydrogen-bond geometry $\left(\AA,{ }^{\circ}\right)$

\begin{tabular}{|c|c|c|c|c|}
\hline$D-\mathrm{H} \cdots A$ & $D-\mathrm{H}$ & $\mathrm{H} \cdots A$ & $D \cdots A$ & $D-\mathrm{H} \cdots A$ \\
\hline $\mathrm{N} 2-\mathrm{H} 2 \cdots \mathrm{N} 6$ & $0.86(2)$ & $2.33(2)$ & $3.176(5)$ & $173(3)$ \\
\hline $\mathrm{N} 5-\mathrm{H} 5 \cdots \mathrm{N} 3$ & $0.85(2)$ & $2.37(2)$ & $3.220(5)$ & $172(4)$ \\
\hline
\end{tabular}

